\title{
Where Do You Look? Visual Attention to Human Bodies across the Weight Spectrum in Individuals with Normal Weight or with Obesity
}

\author{
Elisabeth Leehr ${ }^{a} \quad K^{2}$ atrin Elisabeth Giel ${ }^{a} \quad$ Norbert Schaeffeler ${ }^{a} \quad$ Isabelle Mack ${ }^{a}$ \\ Ansgar Thiel $^{b}$ Guido Zurstiege $^{c} \quad$ Stephan Zipfel ${ }^{a}$ \\ a Department of Psychosomatic Medicine and Psychotherapy, University Hospital Tübingen, \\ Tübingen, Germany; ${ }^{b}$ Institute of Sports Science, Eberhard Karls University Tübingen, Tübingen, \\ Germany; ${ }^{C}$ Institute of Media Science, Eberhard Karls University Tübingen, Tübingen, Germany
}

\section{Keywords}

Attention $\cdot$ BMI $\cdot$ Body $\cdot$ Eye tracking $\cdot$ Obesity

\begin{abstract}
Objective: We investigated how individuals with normal weight (NW) versus individuals with obesity (OB) visually explore human bodies varying in BMI. Methods: 16 OB (BMI $37.5 \pm 7.7$ $\mathrm{kg} / \mathrm{m}^{2}$ ) and $35 \mathrm{NW}\left(\mathrm{BMI} 21.5 \pm 1.6 \mathrm{~kg} / \mathrm{m}^{2}\right.$ ) visually explored 35 natural photographs and 25 avatars depicting male and female bodies ranging from normal weight to obesity. Gaze behaviour was assessed using eye tracking technology. Additionally, participants rated the attractiveness of all body stimuli. Results: A comparison of the displayed gaze behaviour shown by both participant weight classes indicates that regarding visual stimuli of obese natural bodies, all participants $(\mathrm{OB}+\mathrm{NW})$ looked at the waist longer, whereas regarding visual stimuli of normal-weight bodies, all participants viewed the head longer. Overall, OB spent less time looking at the bodies than NW. All participants rated normal-weight bodies as more attractive than obese bodies. However, the NW sample rated the depicted obese bodies as even less attractive than the OB sample did. Conclusions: The BMI of the explored body, but not the observer's BMI, produces differences in the visual exploration of human bodies. The displayed exploration pattern of obese bodies can cautiously be interpreted as a 'de-individualised' and 'obesity-focused' perception. This tendency may prove in close relationship with the negative cultural views on obesity.

(C) 2018 The Author(s)

Published by S. Karger $\mathrm{GmbH}$, Freiburg
\end{abstract}




\section{Introduction}

In modern European societies, obesity is considered norm 'deviant', which causes individuals suffering from obesity to often experience a negative evaluation in public perception [1]. This negative evaluation can also be internalised by individuals with obesity themselves, causing a weight bias internalisation [2] that effects their self-worth concepts. This biased perception may be reflected in the manner with which visual exploration of obese bodies occurs, for example in a specifically selective visual attention to body stimuli [3]. Selective attention to body parts, as a form of the attentional processing of body-related information, might subsequently contribute to the perpetuation of obesity based on reoccurring experiences of body dissatisfaction [4] and perceived weight stigmatisation, which are known to influence weight-related behaviours [5].

Studies show that attention allocation usually focuses on body parts that communicate social information, especially the face (e.g. [6, 7]). However, research on eating disorders shows that a second attention focus on 'index body parts' [8] that communicate information about body shape and size (e.g. waist) occurs as well.

Recent studies have investigated the attentional processing of individuals with eating disorders [8, 9] and obesity [10] of their own bodies. Most of these studies investigated the allocated attention to body parts that participants had previously classified as 'ugly' versus 'beautiful'. These studies have demonstrated that attitudes, such as dissatisfaction towards the body or specific body parts, shape control groups' overall attention to the body. In contrast, individuals with eating disorders spend more time looking at their own 'ugly' body parts [9, 11] or performed upward social comparison patterns [12]. Overall, evidence for an attentional bias in individuals with overweight or obesity is heterogeneous $[9,10]$. A recent review summarised the evidence for heightened body dissatisfaction in individuals with obesity [13].

To date there is little known about the general (excluding self-reference) visual exploration of bodies with varying BMIs ranging from normal weight to obesity. Further, it is not clear whether or not attention allocation to bodies depends on the person's own BMI. Attentional biases in body processing might influence the observer's self-esteem, either increasing or decreasing self-worth [12] and, therefore, influencing subsequent weight-related behaviour [5]. A focused investigation of body stimuli processing and the influence the observer's BMI plays may reveal further attentional biases and further disentangle stigmatisation (attentional bias regarding body stimuli in the overweight/obese spectrum of observers with normal weight) and weight bias internalisation (attentional bias regarding body stimuli in the overweight/obese spectrum of observers with obesity).

In order to investigate the allocation of visual attention to human bodies across the weight spectrum, including individuals with normal weight and individuals with obesity, we developed an eye tracking paradigm. In order to address internal and ecological validity, we compared body stimuli based on natural photographs versus avatars. Avatars are highly standardised computer-generated body stimuli and therefore have the advantage that they are less associated with confounders such as facial expression. Thus, avatars represent body stimuli with high internal validity; however, their high standardisation might also represent a disadvantage in the sense that they are perceived as 'less than real' humans. Therefore, we included a set of natural body photographs. Natural photographs are characterised by high ecological validity and show real human individuals with varying BMI; however, their main disadvantage is that other variables cannot be standardised (e.g. surrounding).

We had the following hypotheses: i) All participants will focus their attention on the face of the depicted persons longer than on other body parts. ii) As obesity and eating disorders share some characteristics $[1,14]$, participants with obesity, when compared to normal- 
Leehr et al.: Where Do You Look? Visual Attention to Human Bodies across the Weight Spectrum in Individuals with Normal Weight or with Obesity

Table 1. Count or means ( \pm standard deviation) of sociodemographic and clinical data of the study sample

\begin{tabular}{lllll}
\hline Variable & $\begin{array}{l}\text { NW } \\
(\mathrm{n}=35)\end{array}$ & $\begin{array}{l}\text { OB } \\
(\mathrm{n}=16)\end{array}$ & $\begin{array}{l}\text { Test statistics } \\
(\text { Chi, tor } \mathrm{U})\end{array}$ & p value \\
\hline Males & 6 & 2 & 0.2 & $>0.05$ \\
Age, years & $23.5(2.7)$ & $25.3(3.9)$ & 194.0 & $>0.05$ \\
BMI, $\mathrm{kg} / \mathrm{m}^{2}$ & $21.5(1.6)$ & $37.5(7.7)$ & 0 & $<0.001$ \\
Lowest self-reported BMI, kg/m ${ }^{2}$ & $19.4(1.3)$ & $26.4(2.7)$ & -8.3 & $<0.001$ \\
Highest self-reported BMI, $\mathrm{kg} / \mathrm{m}^{2}$ & $22.3(1.7)$ & $39.3(10.0)$ & 0 & $<0.001$ \\
ASTS & $45.0(9.5)$ & $47.1(8.9)$ & -0.7 & $>0.05$ \\
EDE-Q: restraint eating & $0.3(0.6)$ & $1.9(1.3)$ & 38.0 & $<0.001$ \\
EDE-Q: eating concern & $0.2(0.4)$ & $0.8(0.7)$ & 90.5 & $<0.001$ \\
EDE-Q: shape concern & $0.6(0.6)$ & $2.5(1.1)$ & 28.0 & $<0.001$ \\
EDE-Q: weight concern & $0.4(0.6)$ & $2.3(0.8)$ & 18.0 & $<0.001$ \\
EDE-Q overall & $0.4(0.4)$ & $1.8(0.8)$ & 18.5 & $<0.001$ \\
EDI-2: drive for thinness & $14.2(5.1)$ & $20.1(5.5)$ & 105.0 & $<0.001$ \\
\hline
\end{tabular}

NW = Controls with normal weight; $\mathrm{OB}$ = sample with obesity; ASTS = Mood Scale, higher values indicating negative mood; EDE-Q = Eating Disorder Examination Questionnaire, higher values indicating higher occurrence of the symptom; EDI-2 = Eating Disorder Inventory, higher values indicating higher drive for thinness.

weight participants, will focus longer on the index parts of obese bodies, which can prove as an indicator for weight bias internalisation. iii) Participants with obesity, when compared to normal-weight participants, will evaluate obese bodies as less attractive.

\section{Material and Methods}

\section{Participants}

16 individuals with a BMI between $30 \mathrm{~kg} / \mathrm{m}^{2}$ and $53 \mathrm{~kg} / \mathrm{m}^{2}$ (OB, 14 females) and 35 individuals with normal weight (NW, 29 females) with a BMI between $19 \mathrm{~kg} / \mathrm{m}^{2}$ and $25 \mathrm{~kg} / \mathrm{m}^{2}$, all between 20 and 35 years, were recruited. Exclusion criteria were non-corrected vision, pregnancy or nursing and an eating disorder assessed via the Eating Disorder Examination Questionnaire (EDE-Q [15], German version [16, 17]). As additional control variables, we assessed mood (ASTS [18]) and the Eating Disorder Inventory subscale 'Drive for thinness' (EDI-2 [19], German Version [20]). Sample characteristics are displayed in table 1.

\section{Stimuli}

We used two sets of stimuli that included female and male bodies depicting normal weight and obesity: to achieve high ecological validity, 35 natural photographs (5 women and 5 men with BMI range 19-24 $\mathrm{kg} / \mathrm{m}^{2}$ and 11 women and 14 men with BMI range $30-49 \mathrm{~kg} / \mathrm{m}^{2}$ ) were included and 25 avatars ( 2 female and 2 male avatars with different BMI, BMI range $19-25 \mathrm{~kg} / \mathrm{m}^{2}$ and $31-36 \mathrm{~kg} / \mathrm{m}^{2}$ ) as highly standardised stimulus material. All stimuli were pre-tested with regard to attractiveness and BMI by experts working in the field of weight-related diseases and eating disorders (table 2). In regard to the rating of attractiveness, the interrater correlation was between $r=0.37$ and $r=0.93$ (all $p<0.05$ ) for natural photographs. In comparison inter-rater correlation was between $r=0.40$ and $r=0.95$ (all $p<0.05$ ) for the avatars. In regard to the BMI rating the inter-rater correlation was between $r=0.69$ and $r=0.95$ (all $p<0.01$ ) for the natural photographs and between $r=0.97$ and $r=0.99$ (all $p<0.01$ ) for the avatars.

\section{Procedure}

The ethics committee of the Medical Faculty at the University Tübingen and the University Hospital Tübingen both approved the study (102/2011B02). All participants gave written informed consent.

Participants in the NW sample were screened via online questionnaires. During the experimental session, all participants filled out questionnaires, and weight and height were assessed in order to calculate 
Leehr et al.: Where Do You Look? Visual Attention to Human Bodies across the Weight Spectrum in Individuals with Normal Weight or with Obesity

Table 2. Results of the stimulus pre-test: Means \pm standard deviation regarding BMI and attractiveness expert rating ${ }^{\mathrm{a}}$

\begin{tabular}{llll}
\hline Stimulus type & Weight class & BMI & Attractiveness \\
\hline \multirow{2}{*}{ Avatar } & normal-weight bodies & $21.6 \pm 1.3$ & $6.4 \pm 0.7$ \\
\multirow{2}{*}{ Natural photographs } & overweight/obese bodies & $32.7 \pm 3.5$ & $3.5 \pm 0.9$ \\
& normal-weight bodies & $21.0 .7 \pm 0.8$ & $6.9 \pm 1.0$ \\
& overweight/ obese bodies & $39.6 \pm 4.3$ & $3.4 \pm 1.1$ \\
\hline
\end{tabular}

aWeight was rated using the BMI; attractiveness was rated on a 1 to 10 scale, 1 indicating very unattractive, 10 indicating very attractive.

BMI. Participants sat in front of a screen and were instructed to freely explore the stimuli as though they were watching television, during this time eye movements were recorded. Each of the 60 trials started with a fixation cross $(1,000 \mathrm{~ms})$ followed by the body picture presented for 4,000 ms. Natural photographs and avatars were presented block-wise in a randomised order. Subsequently, participants rated the stimuli with regard to BMI and attractiveness on a computerised rating scale, ranging from 1 (very unattractive) to 10 (very attractive).

\section{Apparatus}

Eye movements were recorded using the IViewX Hi-Speed eye tracking system (SMI, Berlin, Germany) with a $500 \mathrm{~Hz}$ sampling rate and $0.25-0.5^{\circ}$ gaze position accuracy. Distance between the subject's head and the 19 -inch computer screen with a resolution of $1,280 \times 1,024$ pixels was $60 \mathrm{~cm}$.

\section{Gaze Data}

Gaze data were analysed using BeGaze 3.0 (SMI). Trials were excluded if the fixation cross was not fixated (number of excluded trials over all participants $n=2$ ) or technical problems occurred during data acquisition, e.g. delayed start or early stop of the eye tracking system (number of participants with reduced trial number $\mathrm{n}=2$ ) or bad recording of the eye movement in one condition (number of participants for which data from the avatar condition was excluded $n=3$ ). Corresponding to Hewig et al. [7], we defined seven areas of interest (AOI): head, shoulder, arms, chest, waist, hips, legs. We calculated total dwell time per AOI for normal-weight versus obese body stimuli. Further, the percentage of total dwell time per AOI was used for comparisons.

\section{Statistical Analyses}

Statistical analyses were conducted using SPSS 21 (IBM, Armonk, NY, USA). Only lowest self-reported $\mathrm{BMI}$ and mood scales fulfilled normal distribution criteria and were analysed with t-tests, whereas all other self-report measures were analysed with non-parametric tests (Mann-Whitney Test), since the assumption of normality was violated (age, BMI, highest self-reported BMI, EDE-Q scales, EDI-2 scale). Possible group differences regarding gender were tested with a chi-squared test. Gaze data was analysed separately for natural photographs and avatars using an analysis of variance (ANOVA) with group (NW versus OB) as a between-subject factor and weight class (normal-weight versus obese bodies) and AOI (head, shoulder, arms, chest, waist, hips, legs) as a within-subject factor. Greenhouse-Geisser-corrected values will be reported in the case of sphericity violation. As there was only a small number of men included in the sample, but gender differences are documented regarding body image [21] and the processing of body stimuli [22, 23], we conducted each analyses with and without male participants. The conduction of comparative analyses between genders was not possible because of the small number of male participants. Nevertheless, the inclusion of male participants in our samples did not change results. To investigate the influence of body dissatisfaction on the gaze data we conducted group-wise Pearson correlation analyses between the EDE-Q subscales weight and shape concern and the difference value of dwell time for body stimuli showing obese versus normal weight for each stimulus category. Attractiveness and BMI ratings were analysed using an ANOVA with group as the between-subject factor and weight class as the within-subject factor. For all significant interactions, we conducted post-hoc tests with a Bonferroni-corrected alpha. 
Leehr et al.: Where Do You Look? Visual Attention to Human Bodies across the Weight Spectrum in Individuals with Normal Weight or with Obesity

Table 3. Mean dwell time in \% (standard error) for each AOI of normal weight and obese stimuli

\begin{tabular}{lll}
\hline AOI & Normal-weight bodies & Overweight/obese bodies \\
\hline Avatars & & \\
Head & $21.9(1.4)$ & $20.1(1.6)$ \\
Shoulder & $7.5(0.7)$ & $5.6(0.7)$ \\
Arms & $1.6(0.2)$ & $1.9(0.4)$ \\
Breast & $9.9(0.7)$ & $8.9(0.6)$ \\
Waist & $5.9(0.5)$ & $7.4(0.5)$ \\
Hips & $9.4(0.7)$ & $15.1(1.1)$ \\
Legs & $17.7(1.1)$ & $18.4(1.2)$ \\
\hline Natural photographs & & \\
Head & $28.7(1.6)$ & $24.3(1.3)$ \\
Shoulder & $5.5(0.6)$ & $5.8(0.5)$ \\
Arms & $4.2(0.3)$ & $5.8(0.2)$ \\
Breast & $7.0(0.4)$ & $8.1(0.3)$ \\
Waist & $5.8(0.4)$ & $10.7(0.7)$ \\
Hips & $11.0(0.5)$ & $5.6(0.4)$ \\
Legs & $7.7(0.5)$ & $7.6(0.4)$ \\
\hline
\end{tabular}

$\mathrm{AOI}=$ Area of interest.

\section{Results}

\section{Gaze Data}

Avatars

Main effects were found for weight class, $F(1,45)=10.5, \mathrm{p}<0.001, \eta_{\mathrm{p}}{ }^{2}=0.3$, and AOI, $\mathrm{F}(2.7,120)=49.4, \mathrm{p}<0.001, \eta_{\mathrm{p}}{ }^{2}=0.5$, as well as a significant interaction between these two factors, $\mathrm{F}(3.9,1.74)=13.5, \mathrm{p}<0.001, \eta_{\mathrm{p}}{ }^{2}=0.2$. Post-hoc tests revealed that participants focused on the shoulders of normal-weight bodies for a longer time than on the shoulders of obese bodies, $\mathrm{t}(46)=3.7, \mathrm{p}=0.001, \mathrm{~d}=-0.4$. Dwell time for the index body parts waist, $\mathrm{t}(46)=-3.8, \mathrm{p}=0.001, \mathrm{~d}=0.5$, and hips, $\mathrm{t}(46)=-8.7, \mathrm{p}=0.001, \mathrm{~d}=1.2$, was longer for obese bodies than for normal-weight bodies. Mean percentages of dwell times are displayed in table 3. Correlation analyses revealed no significant correlation between body dissatisfaction scales (EDE-Q-shape and weight concern) and attention allocation (all p > 0.05).

\section{Natural Photographs}

There was a significant group effect, $\mathrm{F}(1,48)=10.2, \mathrm{p}=0.003, \eta_{\mathrm{p}}{ }^{2}=0.2$, showing that the NW sample (mean $=71.6 \%$, SE $=1.5 \%$ ) took a longer look at the bodies in general than did the OB sample (mean $=63.0 \%, \mathrm{SE}=2.4 \%$ ). Analysis revealed a significant main effect of AOI, $\mathrm{F}(1.7,80.6)=97.5, \mathrm{p}<0.001, \eta_{\mathrm{p}}{ }^{2}=0.7$, as well as a significant interaction between AOI and weight class, $\mathrm{F}(3.9,188)=31.3, \mathrm{p}<0.001, \eta_{\mathrm{p}}{ }^{2}=0.4$. Post-hoc tests displayed that participants looked longer at the head, $\mathrm{t}(49)=5.1, \mathrm{p}<0.001, \mathrm{~d}=-0.4$, and the hips, $\mathrm{t}(49)=10.5, \mathrm{p}<0.001$, $\mathrm{d}=-1.4$, of normal-weight bodies than on head and hips of obese bodies. Conversely, all participants examined the arms, $\mathrm{t}(49)=-5.0, \mathrm{p}<0.000, \mathrm{~d}=0.9$, breast, $\mathrm{t}(49)=-3.2, \mathrm{p}=0.002$, $\mathrm{d}=0.4$, and waist, $\mathrm{t}(49)=-7.8, \mathrm{p}<0.001, \mathrm{~d}=1.7$, of obese bodies longer than of normal-weight bodies. Mean percentages of dwell times are displayed in table 3. Correlation analyses revealed no significant correlation between body dissatisfaction scales (EDE-Q-shape and weight concern) and attention allocation (all $p>0.05$ ), except for a significant positive correlation between the EDE-Q-shape concern and the difference value for the AOI head in the OB 
Fig. 1. Mean attractiveness (range from 1 to 10,1 indicating very unattractive, 10 indicating very attractive) and standard error. A Mean attractiveness rating for avatar stimuli. B Mean attractiveness rating for natural stimuli. $\mathrm{NW}=$ individuals with normal weight; $\mathrm{OB}=$ individuals with obesity.

Leehr et al.: Where Do You Look? Visual Attention to Human Bodies across the Weight Spectrum in Individuals with Normal Weight or with Obesity

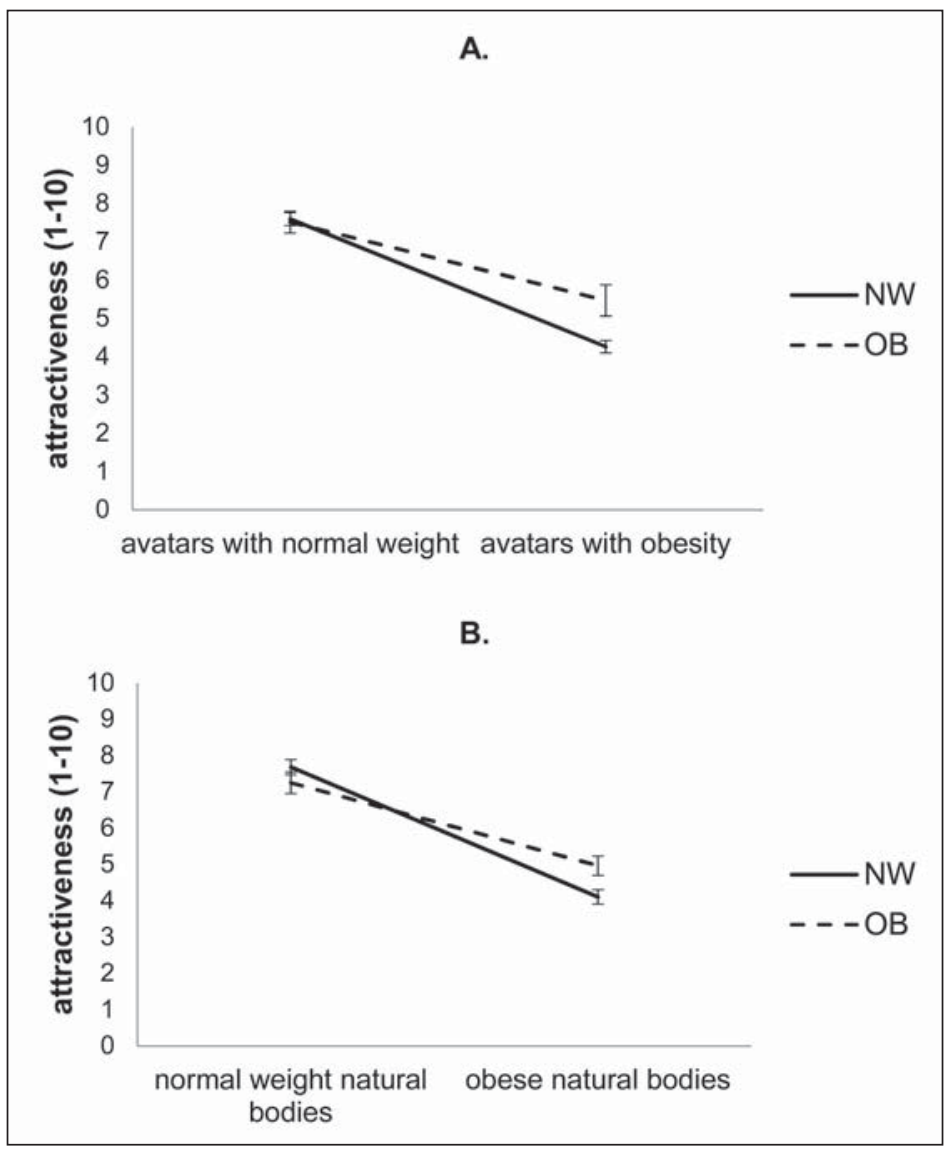

sample, $r=0.7, p<0.01$. High scores regarding shape concern were associated with a higher dwell time for the head of obese bodies when compared to normal-weight bodies.

\section{Attractiveness Rating}

Both groups rated avatars with normal weight as more attractive than avatars with obesity, $\mathrm{F}(1,48)=94.2, \mathrm{p}<0.001, \eta_{\mathrm{p}}{ }^{2}=0.7$ (fig. 1 ). The NW sample rated the attractiveness of both avatars and obese natural bodies as significantly lower than the OB sample: for avatars $\mathrm{F}(1,48)=5.4, \mathrm{p}=0.024, \eta_{\mathrm{p}}{ }^{2}=0.1 ; \mathrm{t}(48)=-3.3, \mathrm{p}=0.002, \mathrm{~d}=1.3$; and for natural bodies $\mathrm{F}(1$, 48) $=5.0, \mathrm{p}=0.03, \eta_{\mathrm{p}}^{2}=0.1 ; \mathrm{t}(48)=-2.5, \mathrm{p}=0.015, \mathrm{~d}=0.5$ (fig. 1).

\section{BMI Rating}

The BMI rating of avatars revealed a significant main effect of weight class, $F(1,48)=$ 498.1, $\mathrm{p}<0.001, \eta_{\mathrm{p}}{ }^{2}=0.9$, and no significant main effect of group, $F(1,48)=0.3, \mathrm{p}=0.586$, $\eta_{\mathrm{p}}{ }^{2}=0.01$. We found a main effect for weight class, $F(1,48)=1301.7, \mathrm{p}<0.001 \eta_{\mathrm{p}}{ }^{2}=1.0$, a significant main effect for group, $\mathrm{F}(1,48)=7.9, \mathrm{p}=0.007, \eta_{\mathrm{p}}{ }^{2}=0.1$, and a significant interaction between weight class and group, $F(1,48)=6.7, p=0.012, \eta_{p}{ }^{2}=0.1$, for the natural photographs. Post-hoc tests displayed significant rating differences between both groups, but only for the obese bodies, $\mathrm{t}(48)=-2.9, \mathrm{p}=0.006, \mathrm{~d}=0.9$, showing that on average the OB sample rated obese bodies with a higher BMI, mean $=32.7 \mathrm{~kg} / \mathrm{m}^{2}, \mathrm{SE}=0.5 \mathrm{~kg} / \mathrm{m}^{2}$, than the $\mathrm{NW}$ sample, mean $=31.0 \mathrm{~kg} / \mathrm{m}^{2}, \mathrm{SE}=0.3 \mathrm{~kg} / \mathrm{m}^{2}$ ). 


\section{Discussion}

We investigated the allocation of visual attention to human bodies across the weight spectrum in individuals with normal weight and individuals with obesity. Our results suggest that the BMI of the explored body, but not the BMI of the observer, influences attention allocation during the processing of body stimuli. The BMI of the explored body produced differences in visual exploration of all participants. Participants observing photographs of individuals with obesity looked longer at an 'index body part' (waist) than in the face of the depicted bodies. Thus, when confronted with obesity, participants were more interested in regions that are indicative of $\mathrm{BMI}$ and represent negative body-related stereotypes than in areas that transport social and individual information. We cautiously interpret this finding as an indication of a 'de-individualised' and 'obesity-focused' approach of perceiving individuals with obesity. This discovered tendency may be in close relationship with the negative cultural viewpoint concerning obesity - as also expressed in the attractiveness ratings of stimuli - and even weight stigmatisation [24-26]. This de-individualisation effect corresponds to results of studies investigating deviant body appearance (e.g. [27, 28]). Both studies found that pictures of individuals with deviant body appearance, e.g. individuals with port-wine stain or strabismus [28] and children with cleft lip [27], were associated with an attentional bias towards viewing them: a physical deviant face appearance attracted more attention without increasing memory for these faces [28], and the mouth area of children with cleft lip was dwelt on longer than viewing children without cleft lip, whereas the eye area of children with cleft lip was fixated less [27].

In contrast to our hypothesis, the overall visual exploration of bodies did not differ between the $\mathrm{OB}$ and NW sample. The BMI of an observer (considering individuals with normal weight and obesity) therefore seems to have hardly any influence on attentional processing of body stimuli. This corresponds to one recent study investigating visual exploration of body stimuli in individuals with underweight and an eating disorder and individuals with normal weight [29]. In contrast, several studies in the field of eating disorders found differences regarding the exploration pattern of body stimuli in anorexia nervosa (e.g. [30-34]) and bulimia nervosa (e.g. [34-37]) compared to healthy controls. One explanation for these inconsistent results might be the fact that BMI-related differences in the exploration pattern of body stimuli are specific for clinically diagnosed groups which are characterised by body image disturbance symptoms. The only observer-BMI-related difference in the current study was that OB spent less time exploring natural body stimuli than normal-weight participants. This could be interpreted as a visual avoidance pattern in order to reduce discomfort [10]. The avoidance of natural stimuli might serve as self-value protection function [38]. Sonneville et al. [39] found that overweight women who underestimated their body weight showed a less disordered eating pattern and a lower risk for depression, which might be an indicator for a self-serving function. Compared to the NW sample, the OB sample rated the attractiveness of obese bodies as less negative, which could also be interpreted as an attempt to regulate discomfort through a less negative appraisal of in-group members. Nevertheless, as already mentioned, all participants rated normal-weight body pictures as more attractive, indicating a similar beauty ideal in both weight groups. Only the OB sample showed a significant correlation between shape dissatisfaction and gaze pattern, indicating that in the case of 'high shape concern' individuals with obesity looked significantly longer at the head region of obese bodies when compared with normal-weight bodies. This contrasts our general effect of preferred attention allocation to index body parts of natural bodies with obesity. This result also contrasts a study in individuals with normal weight that found that participant's body dissatisfaction increases their attentional bias for thinness [40]. Nevertheless, it might again be an attempt of individuals with obesity suffering from high body dissatisfaction to 
avoid the confrontation, especially with body parts indicative of weight. Durso and Latner [41] promoted the term weight bias internalisation, e.g. the internalisation of negative social stereotypes regarding overweight and obesity by individuals suffering from overweight or obesity. Assuming an internalisation of weight bias, the confrontation with obese bodies, but especially with body parts indicative of weight, might be especially threating for individuals suffering from obesity and high body dissatisfaction.

Taking a clinical perspective, our results highlight the importance of attentional biases for body-related information processing in the treatment of individuals with overweight and obesity. Since attentional biases might be changeable [3], it would be interesting to further investigate the possibility of interventions focusing on the modulation of attentional biases and its possible influence on health behaviour.

In regard to the different stimulus types (avatars vs. natural photographs) results are largely comparable. The most prominent difference is that there was no difference in dwell time for the head of obese bodies compared to normal-weight bodies in the avatar condition. The assumption may be made that the face of avatars is in general not that important since participants might not expect to receive valid social information from avatars in the same way they do from natural faces. Nevertheless, avatar heads were also looked at the most frequently when compared to the other AOIs. Furthermore, whereas in the avatar condition the hips and waist of obese bodies were viewed longer when compared to the hips and waist of normalweight bodies., in the natural photograph condition an attentional bias was found for the waist but not the hips of obese bodies. We interpret this as an artefact since the individuals on the natural photographs were 'in action' and not clothed in a standardised manner. Therefore, the differentiation between hips and waist might not have been very exact. This is one of the first studies to investigate the attentional processing patterns of human bodies by individuals with obesity as compared to individuals with normal weight. We have presented standardised and natural stimulus material to heighten the generalisability of our findings. Limitations of the study are: i) weight bias internalisations which may have an influence on attention allocation toward human bodies was not assessed through questionnaires; ii) the stimulus material did not include the participants' own body; iii) we cannot provide information on mechanisms (e.g. strategies or beliefs, social desirability) underlying the exploration patterns of our study samples; and iv) the number of male participants was too small to detect potential gender differences, although gender differences in body image have been shown in other research (e.g. [42]).

In conclusion, the BMI of the explored body, but not the observer's BMI, produced differences in the visual exploration pattern of human bodies. The exploration pattern of obese bodies might cautiously be interpreted as a 'de-individualised' and 'obesity-focused' perception. Obese bodies are seen as less attractive than normal-weight bodies in general. Individuals with normal weight seem to evaluate obese bodies more negatively than do individuals suffering from obesity. A selective attention pattern to index body parts might be an important contributing factor to various maintenance factors within obesity and states of increased overweight, as it promotes stigmatisation (even though indirect through gaze behaviour) and may be interpreted as a sign for weight bias internalisation. Since perceived weight stigmatisation is known to influence weight-related behaviours [5], it may contribute to the maintenance of states of overweight and obesity.

Future research should focus on the underlying mechanisms of attentional biases, e.g. the attentional processing of simultaneously presented bodies (obese vs. normal-weight) and address the possible contribution of external and internal weight biases in the maintenance of states of overweight and obesity. 
Leehr et al:: Where Do You Look? Visual Attention to Human Bodies across the Weight Spectrum in Individuals with Normal Weight or with Obesity

\section{Acknowledgements}

We thank Viola Renner for linguistic revision of the manuscript.

\section{Disclosure Statement}

None of the authors declares a conflict of interest.

\section{References}

1 Schwartz MB, Brownell KD: Obesity and body image. Body Image 2004;1:43-56.

-2 Puhl RM, Moss-Racusin CA, Schwartz MB: Internalization of weight bias: Implications for binge eating and emotional well-being. Obesity (Silver Spring) 2007;15:19-23.

-3 Lane BR, Mulgrew KE, Mahar D, White MJ, Loughnan SA: The effects of priming in a cued dot-probe task on appearance-related attentional biases in women. Int J Eat Disord 2017;50:817-825.

-4 Stice E, Presnell K, Spangler D: Risk factors for binge eating onset in adolescent girls: a 2-year prospective investigation. Health Psychol 2002;21:131-138.

5 Puhl RM, Heuer CA: Obesity stigma: important considerations for public health. Am J Public Health 2010;100: 1019-1028.

6 Itier RJ, Batty M: Neural bases of eye and gaze processing: the core of social cognition. Neurosci Biobehav Rev 2009;33:843-863.

7 Hewig J, Cooper S, Trippe R, Hecht H, Straube T, Miltner W: Drive for thinness and attention toward specific body parts in a nonclinical sample. Psychosom Med 2008;70:729-736.

-8 Horndasch S, Kratz O, Holczinger A, Heinrich H, Honig F, Noth E, Moll GH: 'Looks do matter' - visual attentional biases in adolescent girls with eating disorders viewing body images. Psychiatry Res 2012;198:321-323.

-9 Svaldi J, Caffier D, Tuschen-Caffier B: Attention to ugly body parts is increased in women with binge eating disorder. Psychother Psychosom 2011;80:186-188.

-10 Warschburger P, Calvano C, Richter EM, Engbert R: Analysis of attentional bias towards attractive and unattractive body regions among overweight males and females: an eye-movement study. PLoS One 2015; 10:e0140813.

11 Jansen A, Nederkoorn C, Mulkens S: Selective visual attention for ugly and beautiful body parts in eating disorders. Behav Res Ther 2005;43:183-196.

12 Blechert J, Nickert T, Caffier D, Tuschen-Caffier B: Social comparison and its relation to body dissatisfaction in bulimia nervosa: evidence from eye movements. Psychosom Med 2009;71:907-912.

13 Weinberger NA, Kersting A, Riedel-Heller SG, Luck-Sikorski C: Body dissatisfaction in individuals with obesity compared to normal-weight individuals: a systematic review and meta-analysis. Obes Facts 2016;9:424-441.

14 Faulconbridge LF, Bechtel CF: Depression and disordered eating in the obese person. Current Obes Rep 2014; 3:127-136.

15 Fairburn CG, Beglin S: The assessment of eating disorders: interview or self-report questionnaire? Int J Eat Disord 1994;16:363-370.

16 Hilbert A, Tuschen-Caffier B: Eating Disorder Examination-Questionnaire: Deutschsprachige Übersetzung. Münster, Verlag für Psychotherapie, 2006.

17 Hilbert A, Tuschen-Caffier B, Karwautz A, Niederhofer H, Munsch S: Eating Disorder Examination-Questionnaire. Diagnostica 2007;53:144-154.

18 Dalbert C: Subjektives Wohlbefinden junger Erwachsener: Theoretische und empirische Analysen der Struktur und Stabilität. Zeitschrift für Differentielle und Diagnostische Psychologie 1992;13:207-220.

19 Garner D: Eating Disorder Inventory - 2. Professional Manual. Odessa, FL, PAR-Psychological Assessment Resources, 1991.

20 Paul T, Thiel A: EDI-2. Eating Disorder Inventory-2. Deutsche Version. Göttingen, Hogrefe, 2005.

-21 Buchanan NT, Bluestein BM, Nappa AC, Woods KC, Depatie MM: Exploring gender differences in body image, eating pathology, and sexual harassment. Body Image 2013;10:352-360.

22 Cazzato V, Mele S, Urgesi C: Gender differences in the neural underpinning of perceiving and appreciating the beauty of the body. Behav Brain Res 2014;264:188-196.

23 Nummenmaa L, Hietanen JK, Santtila P, Hyona J: Gender and visibility of sexual cues influence eye movements while viewing faces and bodies. Arch Sex Behav 2012;41:1439-1451.

-24 Cross N, Kiefner-Burmeister A, Rossi J, Borushok J, Hinman N, Burmeister J, Carels RA: Facial attractiveness, weight status, and personality trait attribution: the role of attractiveness in weight stigma. Obes Res Clin Pract 2017;11:377-388.

25 Giel KE, Zipfel S, Alizadeh M, Schaffeler N, Zahn C, Wessel D, Hesse FW, Thiel S, Thiel A: Stigmatization of obese individuals by human resource professionals: an experimental study. BMC Public Health 2012;12:525. 
Leehr et al:: Where Do You Look? Visual Attention to Human Bodies across the Weight Spectrum in Individuals with Normal Weight or with Obesity

26 Ruffman T, O'Brien KS, Taumoepeau M, Latner JD, Hunter JA: Toddlers' bias to look at average versus obese figures relates to maternal anti-fat prejudice. J Exp Child Psychol 2016;142:195-202.

27 Rayson H, Parsons CE, Young KS, Goodacre TE, Kringelbach ML, Bonaiuto JJ, McSorley E, Murray L: Effects of infant cleft lip on adult gaze and perceptions of 'cuteness'. Cleft Palate Craniofac J 2017;54:562-570.

28 Ackerman JM, Becker DV, Mortensen CR, Sasaki T, Neuberg SL, Kenrick DT: A pox on the mind: disjunction of attention and memory in the processing of physical disfigurement. J Exp Soc Psychol 2009;45:478-485.

29 Phillipou A, Rossell SL, Gurvich C, Castle DJ, Troje NF, Abel LA: Body image in anorexia nervosa: body size estimation utilising a biological motion task and eyetracking. Eur Eat Disord Rev 2016;24:131-138.

- 30 Castellini G, Polito C, Bolognesi E, D’Argenio A, Ginestroni A, Mascalchi M, Pellicano G, Mazzoni LN, Rotella F, Faravelli C, Pupi A, Ricca V: Looking at my body. Similarities and differences between anorexia nervosa patients and controls in body image visual processing. Eur Psychiatry 2013;28:427-435.

-31 Pinhas L, Fok KH, Chen A, Lam E, Schachter R, Eizenman O, Grupp L, Eizenman M: Attentional biases to body shape images in adolescents with anorexia nervosa: an exploratory eye-tracking study. Psychiatry Res 2014; 220:519-526.

32 Bauer A, Schneider S, Waldorf M, Braks K, Huber TJ, Adolph D, Vocks S: Selective visual attention towards oneself and associated state body satisfaction: an eye-tracking study in adolescents with different types of eating disorders. J Abnorm Child Psychol 2017;45:1647-1661.

-33 Bauer A, Schneider S, Waldorf M, Cordes M, Huber TJ, Braks K, Vocks S: Visual processing of one's own body over the course of time: evidence for the vigilance-avoidance theory in adolescents with anorexia nervosa? Int J Eat Disord 2017;50:1205-1213.

-34 Vocks S, Busch M, Grönemeyer D, Schulte D, Herpertz S, Suchan B: Neural correlates of viewing photographs of one's own body and another woman's body in anorexia and bulimia nervosa: an fMRI study. J Psychiatry Neurosci 2010;35:163-176.

-35 Mai S, Gramann K, Herbert BM, Friederich H-C, Warschburger P, Pollatos O: Electrophysiological evidence for an attentional bias in processing body stimuli in bulimia nervosa. Biol Psychol 2015;108:105-114.

-36 Albery IP, Wilcockson T, Frings D, Moss AC, Caselli G, Spada MM: Examining the relationship between selective attentional bias for food- and body-related stimuli and purging behaviour in bulimia nervosa. Appetite 2016; 107:208-212.

-37 Carter FA, Bulik CM, Lawson RH, Sullivan PF, Wilson JS: Effect of mood and food cues on body image in women with bulimia and controls. Int J Eat Disord 1996;20:65-76.

-38 Polivy J, Pliner P: 'She got more than me'. Social comparison and the social context of eating. Appetite 2015; 86:88-95.

-39 Sonneville KR, Thurston IB, Milliren CE, Gooding HC, Richmond TK: Weight misperception among young adults with overweight/obesity associated with disordered eating behaviors. Int J Eat Disord 2016;49:937946.

40 Joseph C, LoBue V, Rivera LM, Irving J, Savoy S, Shiffrar M: An attentional bias for thin bodies and its relation to body dissatisfaction. Body Image 2016;19:216-223.

-41 Durso LE, Latner JD: Understanding self-directed stigma: development of the weight bias internalization scale. Obesity (Silver Spring) 2008;16(suppl 2):80-86.

42 Hoffmann S, Warschburger P: Weight, shape, and muscularity concerns in male and female adolescents: predictors of change and influences on eating concern. Int J Eat Disord 2017;50:139-147. 